\title{
Polymer-Analogous Transformations in Triblock Copolymers Containing Polyacrylamide and Poly(ethylene oxide)
}

\author{
Larisa R. Kunitskaya*, Tatyana B. Zheltonozhskaya, Tatyana I. Rechun \\ Faculty of Chemistry, Taras Shevchenko National University of Kyiv, Volodymyrska Street, 64/13, \\ Kyiv 01601, Ukraine \\ Larisa_kunitskaya@ukr.net
}

Keywords: polyacrylamide, polyethylenoxide, aminomethylation, potentiometric titration.

The polymer-analogous conversion of triblock copolymers (TBC) PAAm- $b$-PEO- $b$-PAAm by the aminomethylation reaction in PAAm blocks under the effect of dimethylamine and formaldehyde (Mannich's reaction) was studied. Kinetic investigations of the Mannich's reaction in TBC as compared to that in pure PAAm were performed by potentiometric titration. The existence of two competitive polymer-analogous transformations in PAAm chains of all the (co)polymers such as aminomethylation and alkaline hydrolysis of amide groups was established and discussed.

\section{Introduction}

The micelle-type polymeric nanocontainers are considered as one of the most perspective carriers to realize the targeted delivery of toxic and/or water-insoluble drugs into certain cells of living organisms. It was shown earlier that asymmetric block copolymers contained chemically complementary polyacrylamide and poly(ethylene oxide) or its monomethyl ether (MOPEO- $b$-PAAm and PAAm- $b$-PEO- $b$ PAAm) form micellar structures in the diluted aqueous solutions [1,2]. Such micelles contain hydrophobic complex "core" formed by the hydrogen-bonded PEO and PAAm chains and hydrophilic "corona" of the surplus segments of longer PAAm blocks. The influence of the anticancer agent doxorubicin (DOX) on the micellization process due to its interaction with the copolymer micelles was established. This opened new prospects for using such copolymers as nanocontainers for DOX and other toxic and poorly soluble drugs. As usual, direct drug's delivery is intracellular process. It could be realized if the drug carriers contain specific ligands (or vectors) such as saccharides, peptides, proteins, antibodies etc. [3-7]. Such vectors can recognize corresponding receptors on a cellular surface, interact with them, and penetrate into the intracellular space by the endocytosis pathway [8,9]. The saccharide vector could be input into a micellar carrier formed due to self-assembly of amphiphilic block copolymers via covalent "sewing" to one of the blocks or by adsorption. The first strategy is more promising because it allows controlling the number and position of vectors in a micelle. 
There are two methods to introduce saccharide vectors into block copolymers via covalent bonding: the first one is in situ, which is carried out during a synthesis of the block copolymer, and the second one is ex situ, which consists in a post-functionalization of previously obtained copolymer. Selection of the appropriate method for a concrete block copolymer depends on the chemical nature of its blocks and the main postulate that the vector should be placing in a micellar "corona". To functionalize efficiently the "corona"-forming PAAm chains in the pointed PEO/PAAm block copolymers it was desirable to convert some amide groups into carboxyl- or amine ones using the reactions of polymer-analogous transformations.

In the present work, we studied the aminomethylation reaction in the micelleforming triblock copolymer PAAm- $b$-PEO- $b$ PAAm since the creation of a number of amino groups onto PAAm chains makes possible not only to expand the range of reactions which are used to input the necessary saccharides, for example, via quaternization of tertiary amine groups with halogen saccharides [10], but also to expand the applications of these micellar nanocarriers to encapsulate and delivery of both the drug substances and genetic materials [11].

\section{Results and discussion}

The titration curves for the modified TBC and PAAm samples are shown in Figure 1.
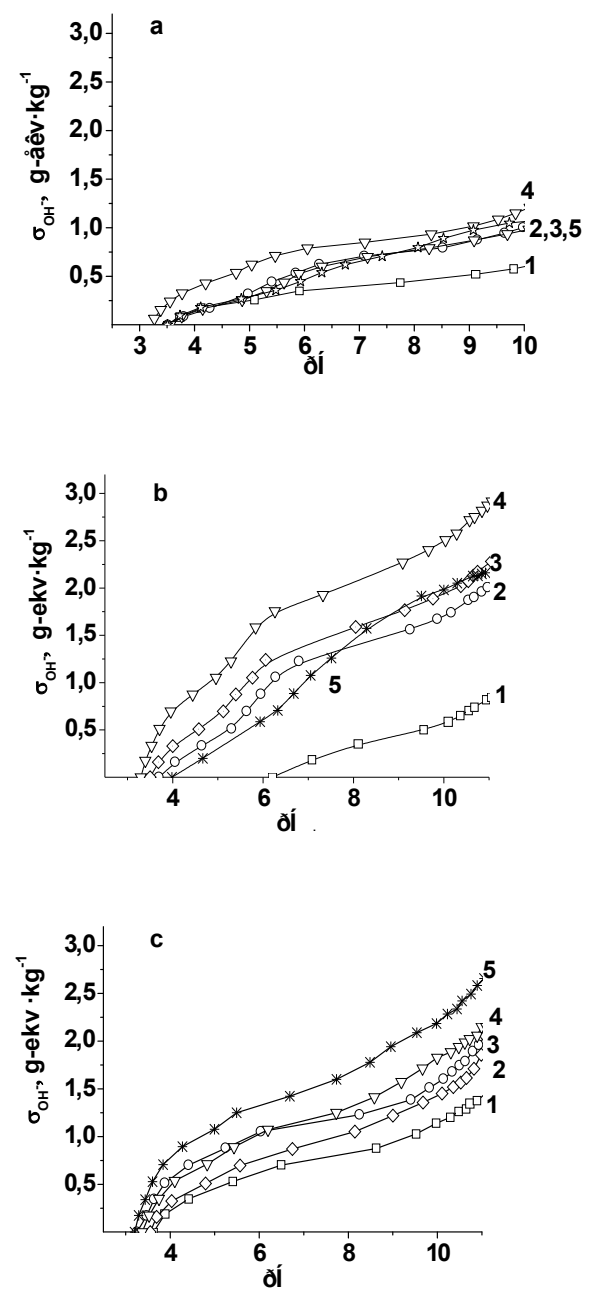

Figure 1. The absorption of hydroxyl ions at different $\mathrm{pH}$ for: (a) TBC1, (b) TBC2, and (c) PAAm in $20-1,60-2$, $120-3,180-4$ and $240 \mathrm{~min}-5$ after aminomethylation beginning.

All the absorption curves demonstrated the areas of so-called "minimal buffer capacity", which are typical for the curves of polyampholytes and pointed out the presence in macromolecules of TBC and PAAm not only amine-, but also carboxyl groups, which are formed by a competitive hydrolysis of some amide groups. Analysis of the results and known data concerning a chemical nature of the main and side products of aminomethylation reaction in polyamides [13] made it possible to 
assume that carboxyl is the first type of groups which are titrated at the alkali addition to the mixture with the Mannich's reaction products (equilibrium 1). The second type of groups, which were titrated after the region of minimal buffer capacity corresponded to the charged tertiary amino groups of aminomethylated chains in TBC and PAAm (equilibrium 2).

$$
\begin{aligned}
& -\mathrm{COOH} \stackrel{\mathrm{K}_{1}}{\longleftrightarrow}-\mathrm{COO}^{-}+\mathrm{H}^{+} \\
& -\mathrm{NR}_{2} \mathrm{H}^{+} \stackrel{\mathrm{K}_{2}}{\longleftrightarrow}-\mathrm{NR}_{2}+\mathrm{H}^{+}
\end{aligned}
$$

These curves allowed defining the limit values of the hydroxyl ion absorption $\left(\sigma_{\lim }\right)$ for each type of groups and the conversion degrees of amide groups into carboxyl- (A) and aminomethyl groups (B) at the polymeranalogous reactions (Figure 2). Practically a stable molar fraction of hydrolyzed links in the aminomethylation products was already reached after $60 \mathrm{~min}$ (Figure 2 a). At the same time, almost permanent value of a molar fraction of the aminomethylated chains was achieved after 20 min (Figure 2 b). Obviously, a significant portion of the unmodified acrylamide units was also present in the solution since the total conversion degree of $\mathrm{A}+\mathrm{B}$ was within 8-18 mol $\%$. These results were expected because the selected reaction conditions would be providing a small degree of PAAm aminomethylation $(\sim 10 \mathrm{~mol} \%)$.
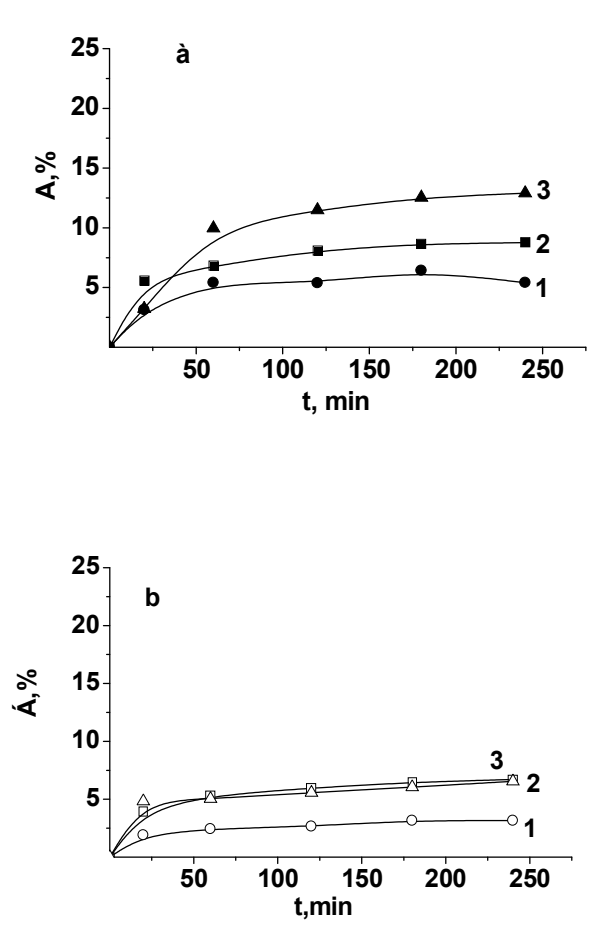

Figure 2. The degrees of: (a) hydrolysis and (b) aminomethylation as functions of the reaction time for TBC $1-1$, PAAm -2 , and TBC2 - 3 .

Parameters of TBC aminomethylation as compared to those for PAAm are shown in Table 2.

Table 2. The kinetic parameters of aminomethylation

\begin{tabular}{|c|c|c|c|c|}
\hline Polymer & $\begin{array}{c}\mathrm{V}_{\mathrm{amin}} \cdot 10^{51)}, \\
\mathrm{mol} \cdot \mathrm{M}^{-3} \cdot \mathrm{c}^{-1}\end{array}$ & $\begin{array}{c}\mathrm{F}^{2)}, \\
\%\end{array}$ & $\begin{array}{c}\mathrm{V}_{\mathrm{hydr}} \cdot 10^{53)} \\
\mathrm{mol} \cdot \mathrm{m}^{-3} \cdot \mathrm{c}^{-1}\end{array}$ & $\begin{array}{c}\mathrm{A}^{4}, \\
\%\end{array}$ \\
\hline PAAm & 3.30 & 6.54 & 4.66 & 8.75 \\
\hline TBC1 & 1.60 & 3.16 & 2.61 & 5.41 \\
\hline TBC2 & 4.19 & 6.71 & 2.69 & 12.89 \\
\hline
\end{tabular}

The rate of aminomethylation. 2) The aminomethylation degree. ${ }^{3)}$ The rate of hydrolysis. ${ }^{4)}$ The hydrolysis degree.

The rates of both the Mannich's and side hydrolysis reactions were calculated to obtain additional data for the aminomethylation process. According to them, TBC2 reacted more intensively than PAAm and TBC1. A significant 
positive role in this process could be attributed to the length of PAAm blocks, which was higher in TBC2. Taking into account the effect of a local concentration of the reacting units and conformation of macromolecules on the kinetics of polymer-analogous reactions in linear polymer chains $[12,13]$ as well as the fact of formation of IntraPCs and following micellar structures with PAAm "coronas" by TBC macromolecules, we could conclude that this effect is related to higher local concentration of active acrylamide units in "coronas" of TBC2 micelles as compared to those of $\mathrm{TBC} 1$ micelles and also to local concentration of acrylamide units in solution of pure PAAm.

\section{Conclusions}

The products of aminomethylation in the triblock copolymers PAAm- $b$-PEO- $b$-PAAm and pure PAAm turned out to be polyampholites that pointed out the proceeding two competitive polymer-analogous transformations in PAAm chains.

The modified TBCs in $60 \mathrm{~min}$ after reaction beginning contained almost twice-increased quantity of carboxyl groups compared to tertiary amine groups. This interesting fact that the reaction of hydrolysis developed more actively than that of aminomethylation in both TBC samples and pure PAAm may be possibly attributed to steric complications. Really, the attacking molecules of dimethylamine and formaldehyde involved in the aminomethylation process are bigger in size than active hydroxyl ions in the alkaline hydrolysis reaction. This can create a certain competition and decrease availability of aminomethylating agents to acrylamide groups. It is believed that simultaneous reactions of hydrolysis and aminomethylation in PAAm chains are possible , although other study devoted to the mechanism of this reaction at different ways of its implementation did not confirm the formation of acrylic acid units.

Taking into account the results of our investigations it is evident that further studies in the field of mechanism of Mannich's reactions in different PAAm-containing polymeric compounds under variable experimental conditions would be continued.

\section{Experimental part}

The initial triblock copolymers $\mathrm{TBC} 1$ and $\mathrm{TBC} 2$ were synthesized by the radical template block copolymerization of PAAm with poly(ethylene glycol) (PEG) initiated by $\mathrm{Ce}^{\mathrm{IV}}$ ions according to the method described earlier [2]. The samples of PEG $\left(M_{n}=6\right.$ and $\left.35 \mathrm{kDa}\right)$ and cerium ammonium nitrate (initiator) from Aldrich (USA) were used for this purpose. The molecular parameters of the obtained block copolymers were determined from ${ }^{1} \mathrm{H}$ NMR spectra recorded in $\mathrm{D}_{2} \mathrm{O}$ at $\mathrm{C}=10 \mathrm{~kg} \cdot \mathrm{m}^{-3}$ and room temperature using a Varian Mercury-400 spectrometer operating at $400 \mathrm{MHz}$. NMR data confirmed the presence of both types of blocks in TBC macromolecules and made it possible to find the molecular weights of PAAm chains and 
whole macromolecules [12]. The results are presented in Table 1.

Table 1. Parameters of the triblock copolymers according to ${ }^{1} \mathrm{H}$ NMR

\begin{tabular}{|c|c|c|c|c|}
\hline Copolymer & $\begin{array}{c}\mathrm{M}_{\mathrm{nPEO}}, \\
\mathrm{kDa}\end{array}$ & $\begin{array}{c}\mathrm{M}_{\mathrm{nPAAm}}{ }^{\mathrm{l})} \\
\mathrm{kDa}\end{array}$ & $\begin{array}{c}\mathrm{M}_{\mathrm{nTBC}}{ }^{2)} \\
\mathrm{kDa}\end{array}$ & $\begin{array}{c}\mathrm{W}_{\mathrm{PEO}}{ }^{3)} \\
\mathrm{w} \%\end{array}$ \\
\hline $\mathrm{TBC} 1$ & 6 & 416 & 422 & 1.42 \\
\hline $\mathrm{TBC} 2$ & 35 & 1897 & 1932 & 1.81 \\
\hline
\end{tabular}

1) The number-average molecular weight of PAAm. ${ }^{2)}$ The molecular weight of TBC: $\mathrm{M}_{\mathrm{nTBC}}=\mathrm{M}_{\mathrm{nPEO}}+2 \cdot \mathrm{M}_{\mathrm{nPAAm}} \cdot{ }^{3)}$ The weight fraction of PEO in the copolymers.

Polymer-analogous transformations in TBC1 and $\mathrm{TBC} 2$ were based on high proton mobility in amide groups that allowed introducing a certain quantity of tertiary amine groups into TBC samples by the aminomethylation reaction with dimethylamine and formaldehyde (Mannich's reaction) according to the scheme:

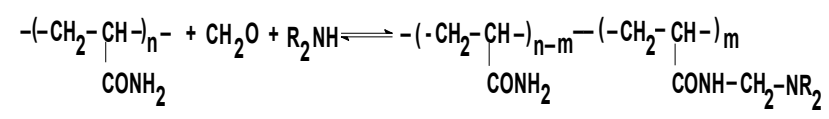

The reaction was carried out at $\mathrm{pH}$ 10.3-10.5 and $\mathrm{T}=50{ }^{\circ} \mathrm{C}$ by the addition of formaldehyde and purified dimethylamine to aqueous solution of TBC $\left(\mathrm{C}=10 \mathrm{~kg} \cdot \mathrm{m}^{-3}\right)$ at a molar ratio of 0.2 : $0.2: 1$. The selected conditions of this reaction as well as the reagent ratios would be ensuring relatively small conversion degree $(\sim 10 \mathrm{~mol} \%)$ of PAAm chains. The solution $\mathrm{pH}$ was adjusted by addition of the appropriate amount of $\mathrm{NaOH}$ $\left(\mathrm{C}_{\mathrm{NaOH}}=1 \mathrm{~mol} \cdot \mathrm{dm}^{-3}\right)$. Under the same conditions the aminomethylation reaction of pure PAAm with $\mathrm{M}_{\mathrm{n}}=117 \mathrm{kDa}$ was carried out. To determine the kinetics of the reaction, the sample splitting was realized in 20,60, 120 and $240 \mathrm{~min}$ after the process beginning. The samples of the obtained cationic derivatives in a salt form were modified in $\mathrm{H}$-form by treating them with $0.5 \mathrm{~N}$ $\mathrm{HCl}$ solution to $\mathrm{pH} \sim 2$. Then they were reprecipitated by acetone, dissolved in water and freeze-dried. Kinetic investigations of the aminomethylation process were performed by potentiometric titration. The modified TBC samples and pure water were titrated using 0.2 $\mathrm{N} \mathrm{NaOH}$. The absorption curves of hydroxyl ions at different $\mathrm{pH}$ values were calculated by the known method described in the study [13].

\section{References}

[1] L.R.Kunitskaya, T.B. Zheltonozhskaya, V.A.Aleinichenko, A.D. Roshal, I.K. Khayetsky, Mol. Cryst. Liq. Cryst . 2011, 536, P.390-404.

[2] T.B. Zheltonozhskaya, et.al. Mol. Cryst. Liq. Cryst . 2011, 536, P.380-391.

[3] T.M.Fahmy, P.M.Fong, A.Goyal, W.M.Saltzman, Materials Today.2005, 8, P.18-26.

[4] A.Mahmud, X.-B.Xiong, M.Aliabadi Hamidreza, A.Lavasanifar, J. Drug Target. 2007, 15, P.553-584

[5] F.Marcucci, F.Lefoulon, Drug Disc. Today, 2004, 9, P.219-228.

[6] M.Shi, J.Lu, M.S.Shoichet, J. Mater. Chem., 2005, 19, P.5485-5498.

[7] D.Sutton, Pharm. Res., 2007, 24, P.1029-1040.

[8] S.Svenson, Am.Chem.Soc., ACS Symp.Ser., 2004, 879, P.2-23.

[9] K.Cho, X.Wang, S.Nie, Z.Chen, D.M.Shin, Clin. Cancer Res., 2008, 14, P.1310-1316.

[10] J.Rieger, F.Stoffelbach, D.Cui, A.Imberty, E.Lameignere, J.L.Putaux, Biomacromolecules, 2007, 8, P.2717-2725. 
[11] Y.Lee, K.Kataoka, Adv. Polym. Sci., 2012, 249, P.95-134.

[12] T. Zheltonozhskaya, E. Shembel, S. Fedorchuk, L. Kunitskaya, I. Maksyuta, N.Permyakova, Y. Gomza. J. of Research Updates in Pol. Sci., 2012, 1, 2, P.1-12.

[13] N.Permyakova, T. Zheltonozhskaya, Y. Poguliai. Mol. Cryst. Liq. Cryst., 2011, 536, P. 372-379. 